\title{
The Plane Wreck's Motion in the Horizontal Direction and the Vertical Direction
}

\author{
Jian Chen \\ North China Electric Power University Baoding
}

Keywords: motion of airframe, wind force, Coriolis Effect

\begin{abstract}
The motion of the lost plane will be affected mainly by the wind stress, and the Coriolis force, of which the Coriolis force will be always on work. The motion of the plane after downed to the water can be divided into three steps. Our model analyzed the plane wreck's motion in the horizontal direction and the vertical direction, respectively, taking the wind force, and the Coriolis Effect into account at the same time.
\end{abstract}

\section{Introduction}

The motion of the airframe will be affected by many factors. So it is sensible to take a detailed analysis on the plane wreck's movement in the seawater. It is beneficial to find out the focused searching areas, arrange the resources such as the search planes, and optimize the scanning routes to find out the lost plane with high efficiency.

\section{Effect of wind stress on plane motion}

To present the effect of the wind [1] on the plane wreck on and under the seawater more clearly, we divide the analysis into three conditions.

\section{A) Floating on the seawater}

The plane will still float on the seawater for a short time before it is entirely submerged. In this case, the wind force will directly act on the airframe. And the velocity of the airframe under the action of the wind can be written as

$$
\left\{\begin{array}{l}
u_{1}=f(v)=a_{1} t_{1} \\
a_{1}=\frac{k_{f} v A \cos \theta-k_{s} V_{c}}{m}
\end{array}\right.
$$

where $v$ is the speed of the wind,

$\boldsymbol{a}_{\mathbf{1}}$ is the acceleration caused by the wind in the horizontal direction,

$\boldsymbol{t}_{\mathbf{1}}$ is the time that the plane keep floating on the seawater (see the following vertical motion analysis section),

$\boldsymbol{k}_{\boldsymbol{f}}$ and $\boldsymbol{k}_{\boldsymbol{s}}$ are respectively the active coefficient of the wind and the resistant coefficient of the seawater,

$\boldsymbol{A}$ is the action area of the wind on the airframe,

$\boldsymbol{\theta}$ is the angle between the wind and the airframe,

and $\boldsymbol{m}$ is the mass of the airframe.

B) Drifting under the seawater

When the airframe is entirely submerged under the seawater, the drifting motion of the plane wreck is of low frequency. In this case, we mainly consider the effect of the wind stress on the energy transport of the low-frequency seawater movement [2]. We suppose that the wind stress is only influenced by its speed and direction on a certain position. The real time information of the wind can be indexed on line from the linking database and then used to modify the effect of the ocean current on the airframe motion. Finally, under the influence of the wind stress on the plane wreck, the velocity of the airframe can be expressed as 


$$
\left\{\begin{array}{l}
u_{2}=f(v, x, y)=a_{2} t_{2} \\
a_{2}=\frac{f_{s 1}(x, y) f_{o} v \cos \beta}{m}
\end{array}\right.
$$

where $\boldsymbol{x}$ and $\boldsymbol{y}$ are respectively the longitude and the latitude to indicate the coordinate of the wind,

$\boldsymbol{a}_{2}$ is the acceleration of the plane caused by the wind,

$\boldsymbol{t}_{\mathbf{2}}$ is the time that airframe drifting under the seawater until arriving the seabed,

$\boldsymbol{f}_{s 1}$ is a coefficient function relating to the geographic location to indicate the effect of the wind on the airframe via indirectly acting on the ocean current,

$f_{0}$ is a coefficient relating to the size and strength of the ocean current,

and $\boldsymbol{\beta}$ is the angle between the wind and the ocean current.

\section{C) Arriving the seabed}

The plane will be falling down in the seawater until it touches the seabed. Once it has arrived at the seabed, it is thought that the plane will be kept stable and the wind will no longer have an effect on the airframe's motion.

\section{Influence of Coriolis force on plane motion}

We also take the Coriolis force [3] into account to obtain a more precise result of the plane motion. The Coriolis force is induced due to the rotation of the coordinate system (the autorotation of the earth). This kind of force will generate an extra Coriolis acceleration which can be written as

$$
a^{k}=2 \omega u
$$

where $\omega$ is the angular velocity of the earth autorotation,

and $u$ is the plane wreck's velocity.

In a period of time, the effect of the Coriolis force on the plane wreck's velocity can be obtained through the following integral performance

$$
\Delta u=\int_{t}^{t+\Delta t} 2 \omega u d t
$$

\section{Horizontal motion of the plane wreck}

Based on the above analysis, we can finally obtain the composite velocity of the plane wreck in the horizontal direction as follows.

1)Floating on the seawater

$$
u_{C}=u_{m}+u_{1}+\Delta u
$$

2)Drifting under the seawater

$$
u_{C}=u_{m}+u_{2}+\Delta u
$$

3)Arriving the seabed

$$
u_{c}=0
$$

where $u_{c}$ is the composite velocity of the aircraft in the horizontal direction.

\section{Analysis on vertical motion of the plane}

Similarly to the former analysis, we also take the study on the vertical motion by three steps. Generally, the main factors that affect the plane wreck's motion in the vertical direction include the wreck's mass $\boldsymbol{m}$, the buoyancy $\boldsymbol{f}_{\mathbf{1}}$, and the resistance $\boldsymbol{f}_{2}$.

Step 1. Floating on the seawater 


$$
\left\{\begin{array}{l}
m g-f_{1}-f_{2}=m a \\
y=\iint a d t^{2} \\
f_{1}=k y \\
f_{2}=k v
\end{array}\right.
$$

where $y$ is the depth of the plane wreck under the seawater,

$\boldsymbol{k}$ is a ratio coefficient of the buoyancy to the depth.

According to parameter of a common aircraft [4], we calculate that the airframe will completely submerged into the seawater with 10 minutes. During this process, the plane wreck is taking a accelerated motion in the vertical direction.

Step 2. Drifting under the seawater

In this process, the buoyancy will be modified, and Eq.(3) reduces to

where $\boldsymbol{\rho}$ is the density of the seawater,

$$
\left\{\begin{array}{l}
m g-f_{1}-f_{2}=m a \\
f_{1}=\rho g V \\
f_{2}=k v
\end{array}\right.
$$

$g$ is the gravity acceleration,

and $\boldsymbol{V}$ is the volume of the plane wreck.

After a short period, the movement of the plane wreck in the vertical direction will be changed from the acceleration motion to a uniform motion[5]. And the detailed velocity during the uniform motion can be calculated by

$$
v=\frac{m g-f_{1}}{k}
$$

\section{Step 3. Arriving at the seabed}

The wreck would stop moving when it touches the seabed.

\section{Composite motion Analysis of the plane}

\section{Step 1. Floating on the seawater}

In this case, we obtain the velocity and the position respectively by

Using the Eq.(7) and Eq.(10) ,we have

$$
u^{2}=\sqrt{u_{c}^{2}+v^{2}}=\sqrt{\left(u_{m}+u_{1}+\Delta u\right)^{2}+v^{2}}
$$

\section{Step 2. Drifting under the seawater}

In this process, the buoyancy will be modified, and Eq.(11) reduces to

Using the Eq.(8) and Eq.(10),we have

$$
u^{2}=\sqrt{u_{c}^{2}+v^{2}}=\sqrt{\left(u_{m}+u_{2}+\Delta u\right)^{2}+v^{2}}
$$

\section{Step 3. Arriving at the seabed}

The wreck would stop moving when it touches the seabed.

\section{Summary}

We index the on-line information of the wind and the ocean current from the linking database to calculate the velocities of the plane wreck in the horizontal direction and the vertical direction, respectively. And then we carry out an integral calculation to obtain the real time position information of the airframe. Further, we take a comparison between the falling depth of the airframe 
and the seabed depth to judge whether the plane has arrived at the seabed. These iterative calculation performances will be kept going until the plane wreck has touched the seabed.

The flow chart is shown as Figure 1.

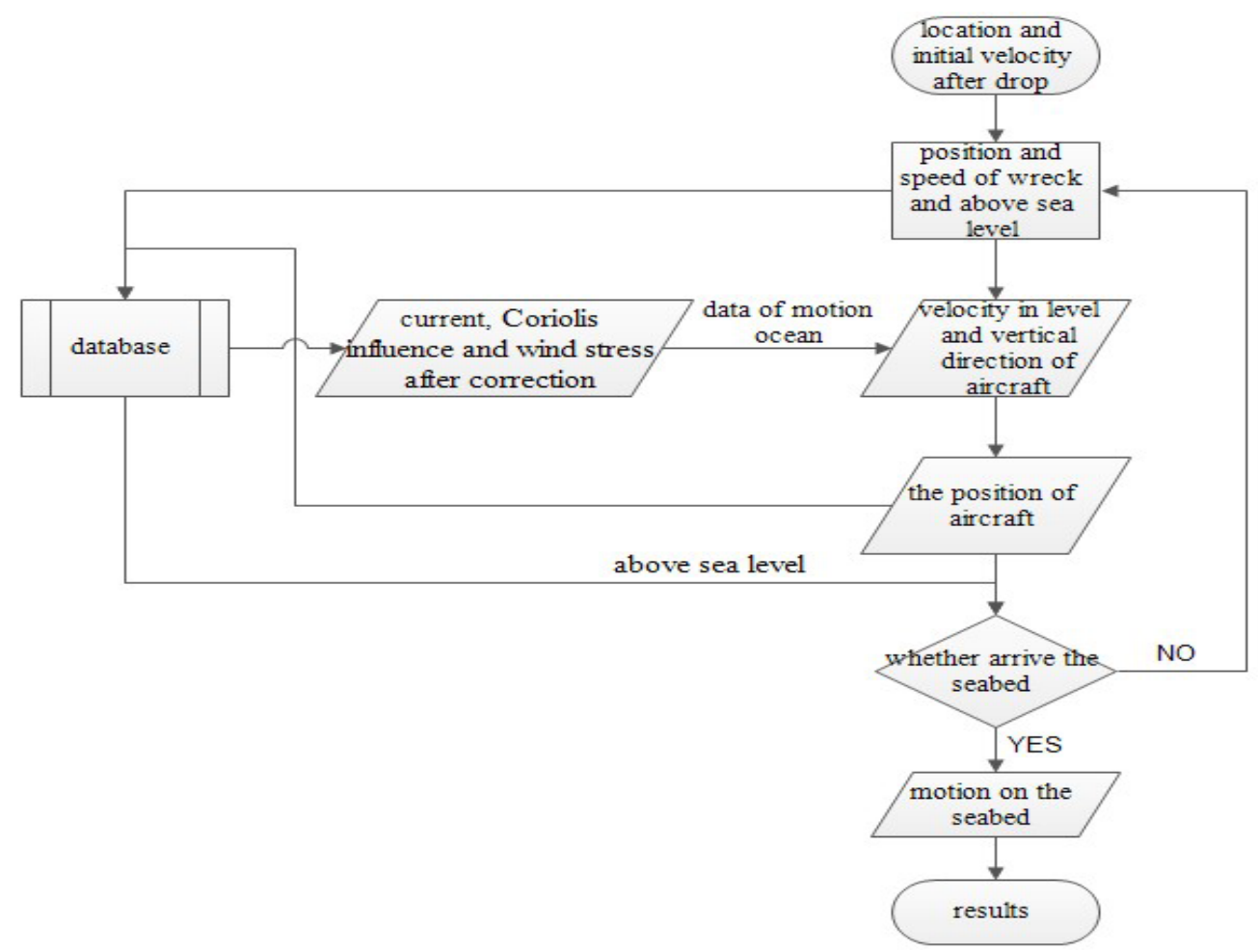

Figure 1. The flow chart

\section{References}

[1]. Marshall, C.F. ; Howard, M.K. ; Guinasso, N.L.,Evaluation of an oil spill trajectory model using satellite-tracked, oil-spill-simulating drifters, Vol.3,2003.

[2]. Information on http://zh.wikipedia.org/wiki/yangliu.

[3]. Xiao-lin Liang, Dissertation Submitted to Shanghai Jiao Tong University for the Degree of Master, 2013.

[4]. Jian-niu Hong, The Deduction of Coriolis Acceleration, 1988.

[5]. Shao-rong Feng, Wen-jun Xiao, An Improved DBSCAN Clustering Algorithm, Journal of China University of Mining\& Technology, Vol.37 No.1, Jan. 2008 